Review

\title{
Insights from Yeast on Oxidative Stress in Alzheimer's Disease, Focusing on Ahp1p/Prx5
}

Ian Macreadie *, Sudip Dhakal

RMIT University, Plenty Road, Bundoora, Victoria 3083, Australia; E-Mails: ian.macreadie@rmit.edu.au; sudip.dhakal@rmit.edu.au

* Correspondence: Ian Macreadie; E-Mail: ian.macreadie@rmit.edu.au

Academic Editor: Michael Fossel

Special Issue: $\underline{\text { Oxidative Stress and Alzheimer's Disease }}$

OBM Geriatrics

2019, volume 3 , issue 3

doi:10.21926/obm.geriatr.1903064
Received: May 05, 2019

Accepted: July 15, 2019

Published: July 17, 2019

\begin{abstract}
The yeast, Saccharomyces cerevisiae, the model eukaryote, has provided much understanding of molecular and cellular biology, as well as insights into many human diseases. In this paper we review how yeast studies are contributing to knowledge about the role of oxidative damage to cell health, and how one of the key players in Alzheimer's disease, amyloid beta $(A \beta)$ is linked to the reactive oxygen species response involving $A H P 1$, which encodes an alkyl hydroperoxidase, Ahp1p, a protein involved in protection from lipid peroxidation.
\end{abstract}

\section{Keywords}

Alkyl hydroperoxidase; Saccharomyces cerevisiae; yeast model for Alzheimer's disease

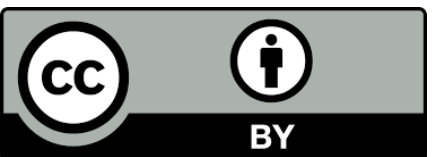

(C) 2019 by the author. This is an open access article distributed under the conditions of the Creative Commons by Attribution License, which permits unrestricted use, distribution, and reproduction in any medium or format, provided the original work is correctly cited. 


\section{Introduction}

There are many factors contributing to Alzheimer's Disease (AD) and oxidative stress is proposed as one such factor [1]. The production of moderate levels of reactive oxygen species (ROS) is an essential and natural process that plays a role in cell signalling. However, ROS is also considered to be a factor in aging [2]. Oxidation can cause damage to lipids, DNA and proteins, which can lead to loss of mitochondrial dysfunction and cause cell death [3]. However, the study of these effects in humans (and in animal models) can be very challenging and limited.

Yeast cells bear many similarities to human cells, including neuronal cells, and for many years yeast have been utilised as a model for Alzheimer's Disease and for studying oxidative stress [4]. One of the attributes of yeast that make it particularly suitable for this research is its ability to be grown by fermentative metabolism even with extensive mitochondrial damage [5]. The outcome of loss of mitochondrial function is that cells no longer have respiratory function and cannot be grown on carbon sources such as ethanol. However, they can still exhibit fermentative growth on fermentable carbon sources such as glucose. Furthermore, ROS accumulation has also been reported to be the major signalling molecules for activation of the genes for pro-inflammatory mediators like cytokines and Nuclear Factor kappa B [6]. It would be intriguing to see how yeast can provide support for studies pertaining to inflammation, specifically in neuroinflammatory diseases.

Many yeast genes that govern important cellular processes are conserved from yeast to humans. Similarities of neurons and yeast cells are not just limited to conserved fundamental processes. Molecules involved in cellular polarization in yeast (during budding and mating) and neurons (during growth of neurons) are structurally and functionally analogous and drive the phenotypic plasticity in these cells [7]. Conserved voltage gated ion channels and other molecules involved in cell-cell communication in yeast cells make them an excellent neuronal model. Formation of yeast flocculation also provides potential for exploring the multicellular dimension that is normal for higher eukaryotes [7].

\section{Pro-Oxidant Chemicals that May be Involved in Alzheimer's Disease}

We are exposed to many chemicals throughout our lives and the effects of the worst of these may be obvious because of their acute toxicity. Chemicals occur naturally in our foods and many are produced by us as well so many have not been subjected to rigorous investigation for their involvement in neurodegenerative disease. Cells make antioxidants such as glutathione and dihydroascorbate as part of their protection against oxidative damage, however, the levels may not always be high enough to provide full protection [8]. Dietary addition can be considered but levels may not reach those required for protection, especially when crossing the blood-brain barrier needs to be considered. Currently evidence that dietary antioxidants provide protective effects in human health is lacking.

In $A D$ the involvement of $A \beta$ [through this manuscript we are referring only to $A \beta(1-42)$ ] is strong and there are links between $A \beta$ and ROS production [9]. In yeast, the constitutive expression of $A \beta$ in the secretory pathway is also implicated in a ROS response: expression of the $A \beta$ caused reduced growth and respiration and increase oxidative stress [2]. In a genome wide expression study of yeast producing cytosolic $A \beta$ fused to GFP (see Figure 1), a stress response was 
observed that indicative of the yeast heat shock response (HSR) [10]. This HSR was confirmed by co-transformation with a plasmid encoding a heat shock reporter: the plasmid contained lacZ downstream of heat shock elements (HSE). In the co-transformants the levels of the enzyme $\beta$ galactosidase were highly elevated by the presence of A $\beta$ fused to GFP [11]. This HSR is likely to be due to the stress caused by ROS, but it should be noted that HSR in yeast can also be triggered by heat shock itself and when protein misfolding is detected.
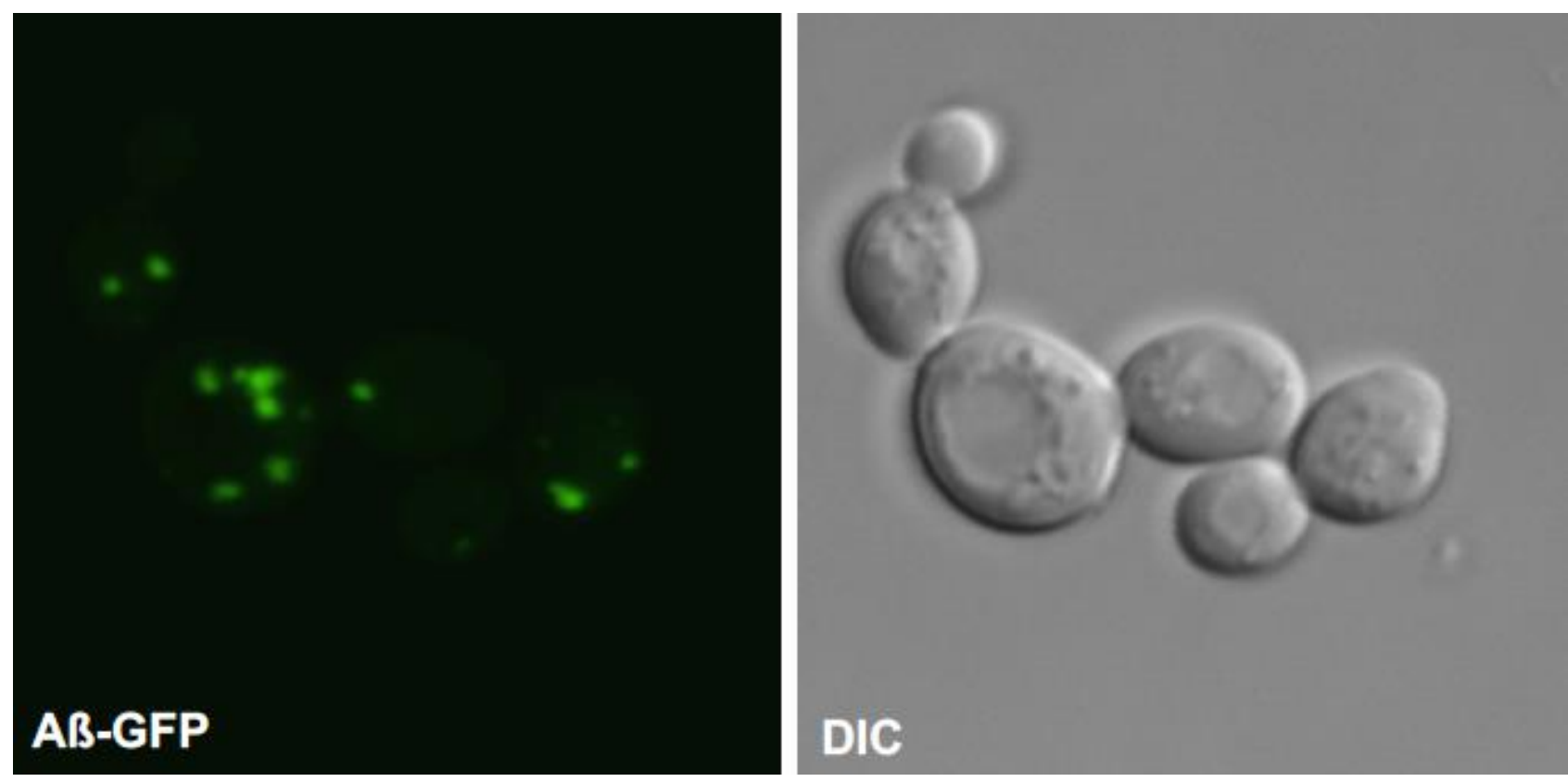

Figure 1 Saccharomyces cerevisiae transformants producing A $\beta$-GFP observed by confocal microscopy and differential interference contrast (DIC) microscopy. Courtesy of Sonia Sankovich, CSIRO.

More recently, using a new yeast reporter system with HSEs upstream of sequences encoding mCherry, red fluorescence was induced by the external addition of chemically-synthesised $A \beta$ peptide. This indicates that extracellular A $\beta$ also causes a HSR [12]. The design of this experiment indicates extracellular $A \beta$ is causing a ROS response.

In addition to the general HSR observed in the genome wide expression analysis, cells producing GFP-A $\beta$ expressed almost twice as much mRNA for AHP1 than those expressing just GFP [10]. This suggested that the encoded alkyl hydroperoxidase is a major contributor used for protection against $A \beta$. Therefore, the use of yeast in screening systems to find chemo preventatives of $A \beta$-induced damage has utilised strains that are deleted for AHP1 [4, 13]. The lack of Ahp1p (the protein encoded by AHP1), increases the sensitivity of these assays [13] so that compounds altering the levels and localisation of $A \beta$ fused to GFP can be more readily ascertained.

The involvement of chemicals as causative agents of $A D$ is currently unclear. We have begun to look for an association by focusing on the effects of biogenic amines that affect mitochondrial respiration. As a start we have looked for, but not found, a synergistic interaction between dopamine and $A \beta$ (Dhakal and Macreadie, unpublished). The effects of other biogenic amines are currently being examined. 


\section{Proteins that May be Involved in Protection against Alzheimer's Disease}

It is also obvious that our genes provide protection against life's stresses. In yeast this has been well studied and the information is readily available in the literature as well as in searches within the Saccharomyces cerevisiae genome database (https://www.yeastgenome.org). In this database the $\sim 6000$ genes of yeast are listed, along with the descriptions of their gene products. The descriptions are systematic, extensive and linked to the relevant literature. Systematic deletions of these genes have led to information about their function and guides the discernment of human orthologs. With regards to protection against ROS the number of genes is considerable. Table 1 provides a list of some yeast genes involved in various modes of protection against ROS and shows their human orthologs that can be identified by sequence similarity. As already mentioned the involvement of Ahp1p in protection in yeast is substantial. This leads to the suggestion that $A \beta$ damage may be via lipid peroxidation. Indeed, this is consistent with the localisation of the 42 amino acid peptide $A \beta$ : it is very hydrophobic and readily localises to membranes. Even the fusion to GFP results in its localisation to "punctate patches" which appear novel (Figure 1). Our current thoughts are inclined to the view that the $A \beta / G F P$ fusion proteins are localised into membranes that have been disrupted from within the cell.

Table 1 Conserved yeast and human genes and encoded proteins conferring protection against Oxidative Damage.

\begin{tabular}{|c|c|c|}
\hline Yeast genes & Encoded proteins & Human orthologs \\
\hline GPX1, GPX2, GPX3/HYR1 & $\begin{array}{c}\text { Phospholipid hydroperoxide } \\
\text { glutathione peroxidase }\end{array}$ & $\begin{array}{c}\text { GPX4: isoform A, isoform } \\
\text { B, isoform C, isoform D }\end{array}$ \\
\hline TSA1, TSA2 & Thioredoxin peroxidase & PRX2 \\
\hline PRX1 (Mitochondria) & Thiol peroxiredoxin & PRX5 \\
AHP1 (Cytoplasm) & Thioredoxin & TRX isoform 1 \\
\hline TRX1, TRX2 (Cytoplasm) & Superoxide dismutase & SOD1 \\
TRX3 (Mitochondria) & SOD2 \\
\hline SOD1 (Cytoplasm) & Glutathione synthase & $\begin{array}{c}\text { Gamma-glutamyl cysteine } \\
\text { synthetase (GCS), GSH }\end{array}$ \\
\hline SOD2 (Mitochondria) & GST \\
\hline GSH1, GSH2 & Omega Class glutathione & Catalase \\
\hline GTO1 (Peroxisome) & transferase & GSR (Mitochondria) \\
\hline GTO3 (Cytoplasm) & Glutathione reductase & Glutathione S transferase \\
\hline CTA1, CTT1 & Glutathione S transferase & \\
\hline GLR1 (Mitochondria) & & \\
\hline GTT1 (ER stress) & & \\
GTT2 (DNA replication stress) & &
\end{tabular}

\section{Ahp1p and its Human Ortholog Prx5: Significance in Neurons}

Following its discovery in 1999, Ahp1p, a peroxiredoxin family protein, has been found to have a significant role in detoxifying the yeast cells from ROS, RNS and alkyl hydroperoxides. 
The protein contains two catalytic cysteines (Peroxidatic Cysteine $\left(C_{P}\right)$ at $C_{y s} 62$ and Resolving Cysteine $\left(C_{R}\right)$ at Cys31), one of which gets oxidized forming cysteine sulfenic acid during the reduction of the oxidized molecules referred to as peroxidatic cysteine and the other one resolves the oxidized cysteine by forming a disulphide bond termed as resolving cysteine [14]. In most cases, cysteines are conserved from Ahp1p to Prx5 (human ortholog of Ahp1p), however some yeast may not have both residues in a monomeric form. S. cerevisiae Ahp1p, existing as a homodimer, requires another molecule of itself to form the cysteine disulphide bond during reduction of the lipid peroxides [14]. In the meantime, Prx5, acts as monomer, has both the residues (Cys47 as $C_{P}$ and $C_{S} 151$ as $C_{R}$ ) within itself and acts independently catalysing the reduction of oxidized lipids (Refer to Figures 2 and 3) [14, 15]. The reduced state of the original molecules is achieved through the help of the NADPH cofactor and enzymes like thioredoxin and thioredoxin reductase [16].

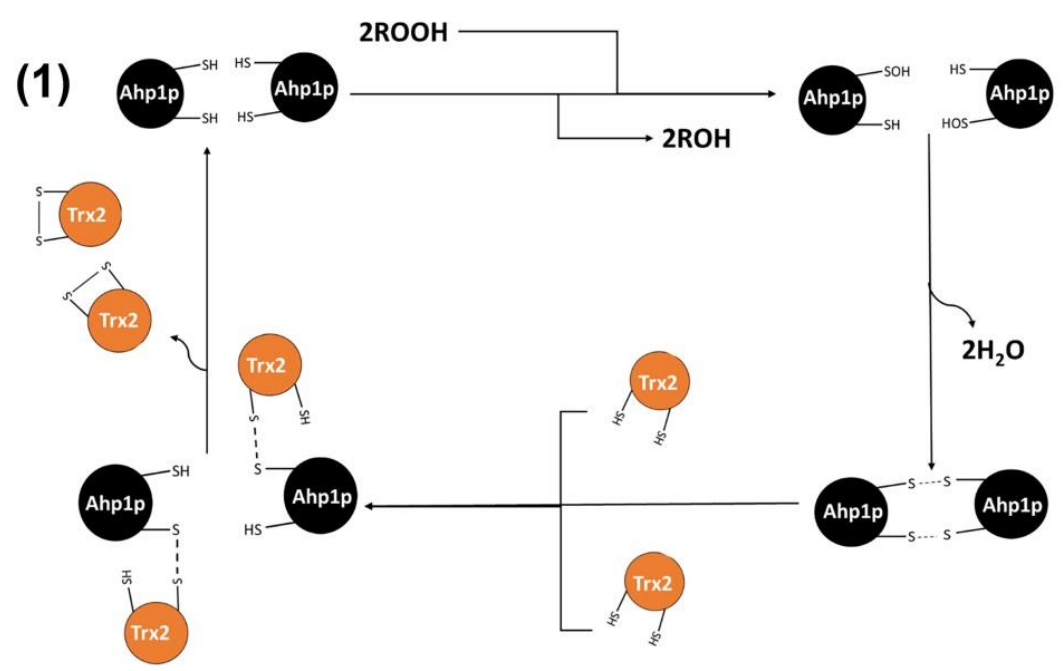

(2)

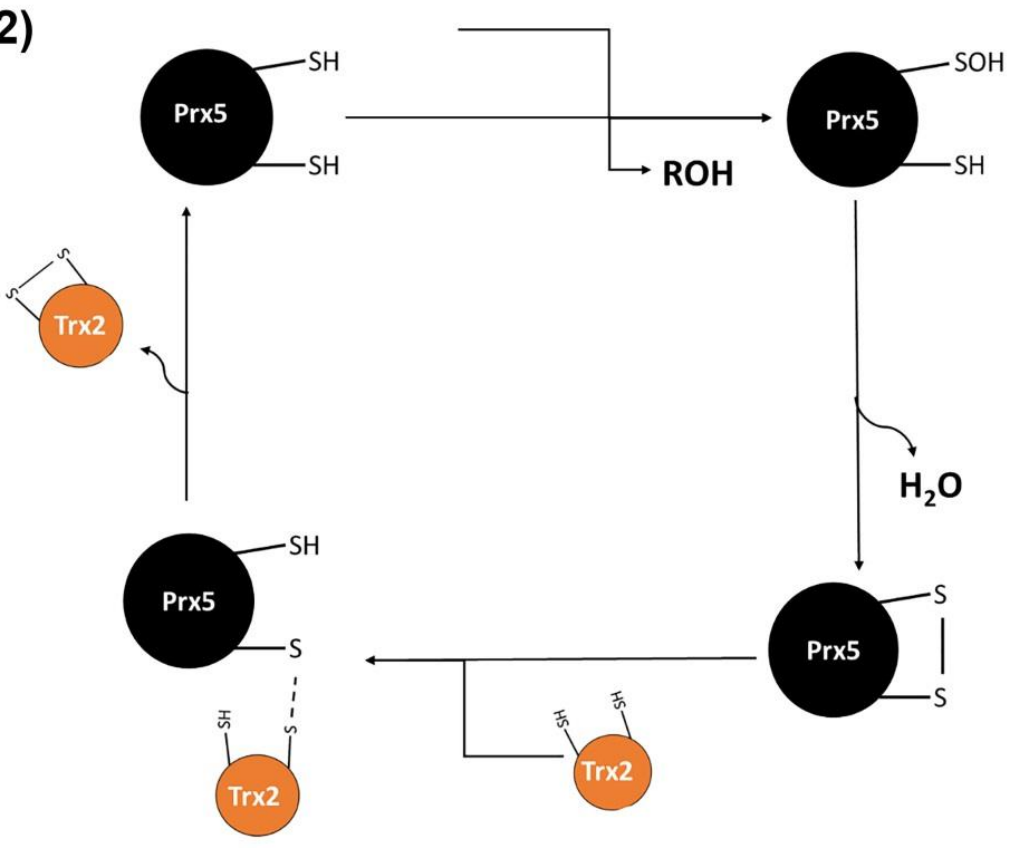

Figure 2 Schematic representation of mechanism of action of Ahp1p (1) and Prx5 (2) against lipid peroxides. 

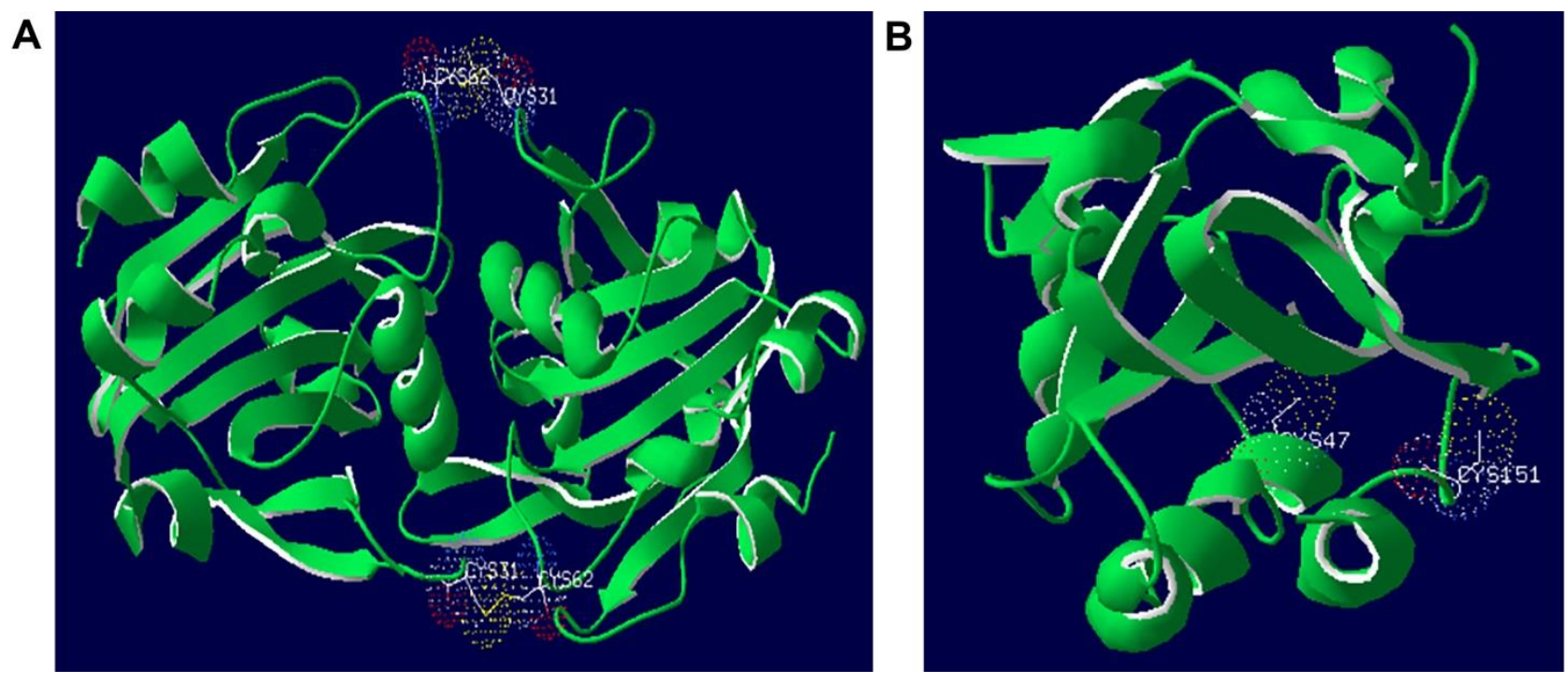

Figure 3 Three-dimensional model for S. cerevisiae Ahp1p (A) and human Prx5 (B) showing conserved cysteine residues.

Among the peroxiredoxin family of proteins in yeast, Ahp1p is a major contributor in providing protection against the metal associated oxidative stress [16]. As shown in Figure 4, the human ortholog of the yeast Ahp1p, peroxiredoxin 5 isoform S, exhibits 30\% sequence identity and $52 \%$ sequence similarity with Ahp1p demonstrating the conserved nature of the Ahp1p. One of the intriguing aspects of human Prx5 is the presence of isoform $L$ which contains a leader peptide (absent in isoform S) that directs it to the mitochondrion. Thus, one gene, through differential transcriptional initiation produced two transcripts. One encodes the $L$ isoform which is a 214 amino acid peroxiredoxin protein that is directed to the mitochondria in humans while the other encodes the shorter 162 amino acid version is located in vacuoles and the cytoplasm.

Multiple functions have been assigned to the human ortholog of Ahp1p from various studies. Some of the important functions of the Prx5 could include down-regulation of the cyclin dependant kinase-5 (Cdk5), clearance of ROS and RNS, protection against oligomeric A $\beta$ associated mitochondrial fragmentation, endoplasmic stress and metal-induced toxicity including iron-induced toxicity represented in Figure 5 [17-20].

Cdk5-p25 complex activation has also been found to be causing hyperphosphorylation of tau that will lead to the formation of neurofibrillary tau tangles in neuronal cells [21]. Considering this evidence from the literature, the protection of the Prx 5 could possibly be one of the crucial events in protecting cells from oxidative stress including in the ageing neuronal cells, which will otherwise deteriorate with accumulation of ROS and misfolded proteins. Studies in cancer cells also highlight the positive effect (to the cancer cells) of overexpression of Prx5 in proliferation and tumorigenicity [22]. This could possibly align with the neuronal cell cycle re-entry hypothesis, where the neuronal cells are triggered to enter the cell division cycle causing the death of the neuronal cells: normally neuronal cells are in a $\mathrm{G}_{0}$ cell cycle and do not divide. Instead, entry into cell division cycle means proceeding towards the death of the cell causing the neuronal loss [23]. 
1 MGLAGVCALRRSAGYILVGGAGGQSAAAAARRYSEGEWASGGVRSFSRAA

1 MSDL-VNKRFPAGDYKFQYIAISQSDADSESCKMPQTVEWSKLISENK

|: .: | ...||

: . : : . : . : :

|..: : : : : $\mid$

51 AAMAPIKVGDAIPA-------VEVFEGEPGNK-------VNLAELF-KGK

48 KVIITGAPAAFSPTCTVSHIPGYINYLDELVKEKEVDQVIVVTVDNPFAN

$|.::| .||.|:| .|: .:|:||:: \ldots|.:| .|.| \ldots|\ldots::|:: . \mid \ldots$

86 KGVLFGVPGAFTPGCSKTHLPGFVEQA-EALRAKGVQVVACLSVNDAFVT

QAWAKSLGVKDTTHIKFASDPGCAFTKSIGFELAVGD------GVYWSGR

.. . : :

$$
.|\ldots: \ldots:| \ldots \mid \text { : . } \mid \text {. . . }
$$

$: \mid .:$.

$|\ldots \ldots|$

135 GEWGRA--HRAEGKVRLLADPTGAFGKET--DLLLDDSLVSIFGNRRLKR

142 WAMVVENGIVTYAARETNP-GTDVTVSSVESVLAHL *

Figure 4 Alignment of the Ahp1p protein (S. cerevisiae S288c) with the human peroxiredoxin 5, mitochondrial protein precursor protein, isoform L (NP 036226.2) of 214 amino acids. A second variant of the human peroxiredoxin 5, isoform $\mathrm{S}$, (NM 001358516.1) results from internal transcription start site, producing a form that lacks the first 52 amino acids and is underlined above. Conserved cysteines are highlighted.

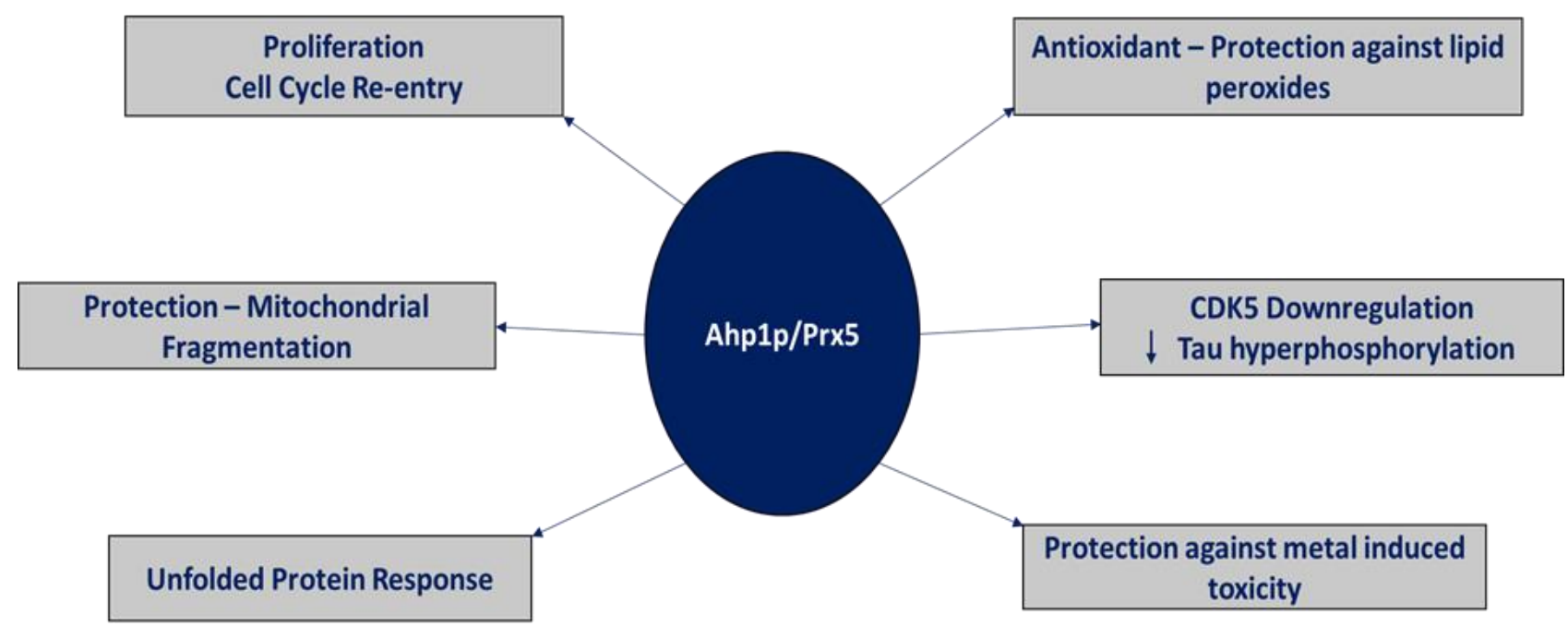

Figure 5 Representation of predicted roles of Ahp1p/Prx5 from various studies.

\section{Conclusions}

Yeast offers a convenient system to dissect the mechanisms in biology, including the understanding of neurodegenerative disease. Here we have presented an analysis of the yeast cellular response to the presence of $A \beta$, which demonstrates a lipid peroxide response. Further studies will address how $A \beta$ causes this particular response and whether there may be other 
factors, such as biogenic amines, drugs and food products, that exacerbate or ameliorate the effects of $A \beta$. The use of yeast enables this work to be performed with relative ease.

\section{Author Contributions}

IGM and SD contributed equally to the conception, research and writing of this article.

\section{Funding}

This research received no specific funding. SD is supported by an RMIT University Ph D Scholarship.

\section{Competing Interests}

The authors have declared that no competing interests exist.

\section{References}

1. Sharma P, Srivastava P, Seth A, Tripathi PN, Banerjee AG, Shrivastava SK. Comprehensive review of mechanisms of pathogenesis involved in Alzheimer's disease and potential therapeutic strategies. Prog Neurobiol. 2019; 174: 53-89.

2. Chen $X$, Petranovic D. Amyloid- $ß$ peptide-induced cytotoxicity and mitochondrial dysfunction in yeast. FEMS Yeast Res. 2015; 15.

3. Pimentel C, Batista-Nascimento L, Rodrigues-Pousada C, Menezes RA. Oxidative stress in Alzheimer's and Parkinson's diseases: Insights from the yeast Saccharomyces cerevisiae. Oxid Med Cell Longev. 2012.

4. Porzoor A, Macreadie I. Yeast as a model for studies on A $\beta$ aggregation toxicity in Alzheimer's disease, autophagic responses, and drug screening. Systems Biology of Alzheimer's Disease (Castrillo JI and Oliver SG ed.), Humana Press; 2015. p. 217-226.

5. Macreadie IG, Bartone N, Sparrow L. Inhibition of respiratory growth and survival in yeast by dopamine and counteraction with ascorbate or glutathione. J Biomol Screen. 2010; 15: 297301.

6. Mittal M, Siddiqui MR, Tran K, Reddy SP, Malik AB. Reactive oxygen species in inflammation and tissue injury. Antioxid Redox Signal. 2014; 20: 1126-1167.

7. Sarto-Jackson I, Tomaska L. How to bake a brain: Yeast as a model neuron. Curr Genet. 2016; 62: 347-370.

8. França MB, Lima KC, Eleutherio ECA. Oxidative stress and amyloid toxicity: Insights from yeast. J Cell Biochem. 2017; 118: 1442-1452.

9. Aleardi AM, Benard G, Augereau O, Malgat M, Talbot JC, Mazat JP, et al. Gradual alteration of mitochondrial structure and function by $\beta$-amyloids: Importance of membrane viscosity changes, energy deprivation, reactive oxygen species production, and cytochrome c release. J Bioenerg Biomembr. 2005; 37: 207-225.

10. Antony $\mathrm{H}$. Study of cellular responses and protein interactions of Abeta in yeast models. Doctor of Philosophy (PhD). Applied Sciences, RMIT University; 2008. 
11. Caine J, Sankovich S, Antony H, Waddington L, Macreadie $P$, Varghese J, et al. Alzheimer's A $\beta$ fused to green fluorescent protein induces growth stress and a heat shock response. FEMS Yeast Res. 2007; 7: 1230-1236.

12. Luu YN, Macreadie I. Development of convenient system for detecting yeast cell stress, including that of amyloid beta. Int J Mol Sci. 2018; 19.

13. Porzoor A, Alford B, Hügel HM, Grando D, Caine J, Macreadie I. Anti-amyloidogenic properties of some phenolic compounds. Biomolecules. 2015; 5: 505-527.

14. Lian FM, Yu J, Ma XX, Yu XJ, Chen Y, Zhou CZ. Structural snapshots of yeast Alkyl Hydroperoxide Reductase Ahp1 peroxiredoxin reveal a novel two-cysteine mechanism of electron transfer to eliminate Reactive Oxygen Species. J Biol Chem. 2012; 287: 17077-17087.

15. Declercq JP, Evrard C, Clippe A, Stricht DV, Bernard A, Knoops B. Crystal structure of human peroxiredoxin 5, a novel type of mammalian peroxiredoxin at $1.5 \mathrm{~A}$ resolution. J Mol Biol. 2001; 311: 751-759.

16. Prouzet-Mauléon V, Monribot-Espagne C, Boucherie H, Lagniel G, Lopez S, Labarre J, et al. Identification in Saccharomyces cerevisiae of a new stable variant of alkyl hydroperoxide reductase 1 (Ahp1) induced by oxidative stress. J Biol Chem. 2002; 277: 4823-4830.

17. Kim B, Park J, Chang KT, Lee DS. Peroxiredoxin 5 prevents amyloid-beta oligomer-induced neuronal cell death by inhibiting ERK-Drp1-mediated mitochondrial fragmentation. Free Radic Biol Med. 2016; 90: 184-194.

18. Lee DG, Kam MK, Kim KM, Kim HS, Kwon OS, Lee HS, et al. Peroxiredoxin 5 prevents iron overload-induced neuronal death by inhibiting mitochondrial fragmentation and endoplasmic reticulum stress in mouse hippocampal HT-22 cells. Int J Biochem Cell Biol. 2018; 102: 10-19.

19. Kim MH, Park SJ, Kim JH, Seong JB, Kim KM, Woo HA, et al. Peroxiredoxin 5 regulates adipogenesis-attenuating oxidative stress in obese mouse models induced by a high-fat diet. Free Radic Biol Med. 2018; 123: 27-38.

20. Park J, Kim B, Chae U, Lee DG, Kam MK, Lee SR, et al. Peroxiredoxin 5 decreases Beta-amyloidmediated Cyclin-Dependent Kinase 5 activation through regulation of $\mathrm{Ca}^{2+}$-mediated calpain activation. Antioxid Redox Signal. 2017; 27: 715-726.

21. Moosavi B, Mousavi B, Macreadie IG. Yeast model of Amyloid- $\beta$ and Tau aggregation in Alzheimer's disease. J Alzheimers Dis. 2015; 47: 9-16.

22. Kim B, Kim YS, Ahn HM, Lee HJ, Jung MK, Jeong HY, et al. Peroxiredoxin 5 overexpression enhances tumorigenicity and correlates with poor prognosis in gastric cancer. Int J Oncol. 2017; 51: 298-306.

23. Koseoglu MM, Norambuena A, Sharlow ER, Lazo JS, Bloom GS. Aberrant neuronal cell cycle reentry: the pathological confluence of Alzheimer's disease and brain insulin resistance, and its relation to cancer. J Alzheimers Dis. 2019; 67: 1-11. 


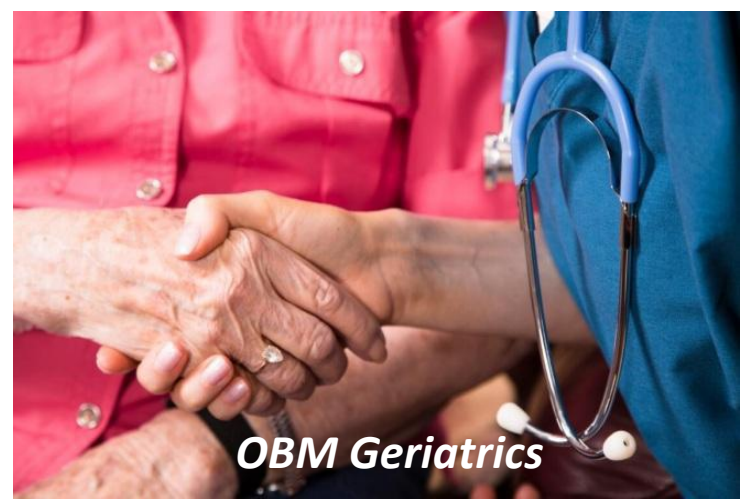

Enjoy $O B M$ Geriatrics by:

1. Submitting a manuscript

2. Joining in volunteer reviewer bank

3. Joining Editorial Board

4. Guest editing a special issue

For more details, please visit: http://www.lidsen.com/journals/geriatrics 